\title{
The effect of a proton pump inhibitor on bone metabolism in ovariectomized rats
}

\author{
MOON KYUNG JOO, JONG-JAE PARK, BEOM JAE LEE, JI HOON KIM, JONG EUN YEON, \\ JAE SEON KIM, KWAN SOO BYUN and YOUNG-TAE BAK
}

\author{
Division of Gastroenterology, Department of Internal Medicine, Korea University \\ College of Medicine, Guro Hospital, Seoul 152-703, Republic of Korea
}

Received October 30, 2012; Accepted February 13, 2013

DOI: $10.3892 / \mathrm{mmr} .2013 .1327$

\begin{abstract}
Recent studies revealed that long-term intake of proton pump inhibitor (PPI) increases the risk of vertebral or hip fracture; however, the exact mechanism for this is not known. To evaluate the effect of long-term PPI therapy on bone turnover, we analyzed the signaling pathway involved in osteoclast differentiation and bone resorption/ formation markers using ovariectomized rats. Six-week-old Sprague-Dawley (S-D) rats were ovariectomized, and two weeks later they were divided into four groups (group A, normal diet + placebo; group B, low calcium diet + placebo; group $\mathrm{C}$, normal diet $+\mathrm{PPI}$; and group $\mathrm{D}$, low calcium diet + PPI). Omeprazole, at a concentration of $30 \mathrm{mg} / \mathrm{kg}$, was administered orally for eight weeks and the rats were sacrificed when they were 16 weeks old. The relative expression levels of the receptor activator of $\mathrm{NF}-\kappa \mathrm{B}$ ligand (RANKL)/osteoprotegerin (OPG) ratio, c-Fos, nuclear factor of activated T cells c1 (NFATc1) and osteocalcin in femoral bone marrow cells were compared, and serum C-terminal crosslinking telopeptide of type I (CTX-1) levels were determined. The relative ratio of RANKL/OPG was increased in group D, and gene expression levels of c-Fos and NFATc1 were upregulated in groups $\mathrm{B}$ and $\mathrm{D}$, which are involved in differentiation and activation of osteoclasts. Furthermore, expression levels of osteocalcin, a bone formation marker, were decreased and levels of serum CTX-1, a bone resorption marker, were
\end{abstract}

Correspondence to: Dr Jong-Jae Park, Division of Gastroenterology, Department of Internal Medicine, Korea University College of Medicine, Guro Hospital, 148 Gurodong-Ro, Guro-Dong, Guro-Ku, Seoul 152-703, Republic of Korea

E-mail: gi7pjj@yahoo.co.kr

Abbreviations: GERD, gastroesophageal reflux disorder; PPI, proton pump inhibitor; NSAID, non-steroidal anti-inflammatory drug; V-ATPase, vacuolar-ATPase; RANKL, receptor activator of NF- $\mathrm{B}$ ligand; OPG, osteoprotegerin; OCP, osteoclast precursor; BMD, bone marrow density; NFATc1, nuclear factor of activated T cell c1; CTX-1, crosslinking telopeptide of type 1; RT-PCR, reverse transcriptase-polymerase chain reaction; ALP, alkaline phosphatase

Key words: proton pump inhibitor, bone metabolism, osteoclast increased in group D. Taken together, a low calcium diet and PPI administration are thought to collaborate in order to alter osteoclast activity and bone resorption signaling.

\section{Introduction}

Gastroesophageal reflux disorder (GERD) is a common disease worldwide, whose prevalence has been on the increase in Eastern Asia in addition to Western countries (1). Accordingly, the prescription of proton pump inhibitors (PPIs) is increasing rapidly (2-5). Long-term PPI therapy has been established as a standard treatment strategy to manage refractory GERD, which has not responded to the standard dose, or recurrent GERD after the withdrawal of PPI treatment (6,7). PPIs have been known to be safe drugs without any major complications with long-term use (8). However, a large-scale epidemiologic study reported that high-dose, long-term therapy with PPIs significantly increased the risk of osteoporosis and pathologic hip fracture (9). A previous nested case-control study revealed that more than one year of PPI therapy is associated with increased risk of hip or femur fracture (10). However, there have been few studies undertaken to clarify the exact mechanism of the PPI effect. It has been established that PPIs reduce the production and secretion of hydrochloric acid in the human stomach, increase the $\mathrm{pH}$ in the stomach or small intestine and inhibit absorption of insoluble calcium in the small intestine, thus leading to pathologic fractures $(11,12)$. Alternatively, several in vitro studies have reported that PPIs inhibit the vacuolar-ATPase (V-ATPase) of osteoclasts and reduce their activity $(13,14)$. Clinical studies have supported the theory that the short-term use of PPI in children or adults does not alter or reduce bone resorption markers $(15,16)$. There are conflicting data regarding the effect of PPI on bone metabolism, however, little is known about the long-term effect of PPI on osteoclast or bone resorption in human or animal models.

Receptor activator of NF- $\kappa \mathrm{B}$ ligand (RANKL), its receptor RANK, and its decoy receptor osteoprotegerin (OPG) are novel concepts in bone metabolism (17). RANKL, a member of the tumor necrosis factor (TNF) family, is a membrane-bound cytokine, and is bound to RANK, a homotrimeric transmembrane protein member of the TNF receptor superfamily, and is expressed on the surface of the osteoclast precursor (OCP). RANKL/RANK binding differentiates the OCP, induces 
Table I. Primer sequence for RT-PCR.

\begin{tabular}{llll} 
Gene & & \multicolumn{1}{c}{ Primer sequence } & Size (bp) \\
\hline RANKL & Sense & 5'-AGCCGAGACTACGGCAAGTA-3' & \\
& Antisense & 5'-GCGCTCGAAAGTACAGGAAC-3' & 208 \\
OPG & Sense & 5'-CTGCCTGGGAAGAAGATCAG-3' & 226 \\
& Antisense & 5'-TTGTGAAGCTGTGCAGGAAC-3' & 247 \\
c-Fos & Sense & 5'-CCAGTCAAGAGCATCAGCAA-3' & 225 \\
NFATc1 & Antisense & 5'-AAGTAGTGCAGCCCGGAGTA-3' & 225 \\
Osteocalcin & Sense & 5'-GGGTCAGTGTGACCGAAGAT-3' & \\
& Antisense & 5'-GGAAGTCAGAAGTGGGTGGA-3' & 157 \\
\hline
\end{tabular}

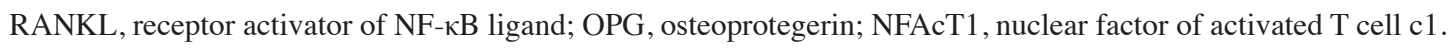

formation, differentiation and activation of osteoclasts, and acts as an essential stimulator for bone resorption. By contrast, OPG is known to inhibit differentiation and activation of osteoclasts effectively under in vivo or in vitro conditions $(18,19)$. As a result, regulation of RANKL or OPG expression is crucial in bone metabolism, and the RANKL/OPG ratio changes in favor of abnormal osteoclastogenesis and is known as a predictive risk factor for pathologic fractures $(20,21)$. Furthermore, sequential activation of $\mathrm{c}-\mathrm{Fos}$ and the nuclear factor of activated $\mathrm{T}$ cell $\mathrm{cl}$ (NFATc1) by the RANKL/RANK/NF- $\kappa \mathrm{B}$ signaling system is essential for the completion of the differentiation process of the OCP and osteoclasts (22-24).

We performed a pilot animal study using ovariectomized ICR mice, into which we injected pantoprazole via an intraperitoneal route, and observed that messenger RNA (mRNA) expression levels of IL-1 $\beta$ were inhibited significantly in the pantoprazole group compared with the control group. No significant difference was observed in the bone marrow density (BMD) between the two groups, from which we hypothesized that the ingestion of PPI can affect bone resorption and bone metabolism (25). In the present study, we aimed to evaluate the long-term effect of PPIs on bone resorption and remodeling in an ovariectomized rat model by analyzing the RANKL/OPG ratio, c-Fos, NFATc1 and osteocalcin, a well-known bone formation biochemical marker, by using bone marrow cells and serum C-terminal cross-linking telopeptide of type I (CTX-1), as a bone resorption marker.

\section{Materials and methods}

Experimental animals. Five-week-old female Sprague-Dawley (S-D) rats were housed for one week for preparatory adaptation to the new environment, ovariectomized at 6 weeks old and allowed to adapt to the inflammatory process for 2 weeks. All the rats were maintained in a laboratory at a temperature of $23 \pm 1^{\circ} \mathrm{C}$ and humidity of $55 \pm 5 \%$ under a 12 -h light-dark cycle (light 7:00-19:00). At 8 weeks old, the animals were subdivided equally into 4 groups. The rats were fed on a regular diet with placebo (group A), low calcium diet with placebo (group B), regular diet with PPI (group C) and low calcium diet with PPI (group D). Solid feed was used for the rats including calcium
$1.25 \%$, phosphorus $0.95 \%$, vitamin D 340 IU/100 mg (Samyang Co., Ltd., Seoul, Korea) as the regular diet, and another solid rat feed including calcium $0.01 \%$, phosphorus $0.95 \%$, vitamin D 340 IU/100 mg (Oriental Yeast Co., Ltd., Tokyo, Japan) for the low calcium diet. Omeprazole was administered orally at a dose of $30 \mathrm{mg} / \mathrm{kg} / \mathrm{day}$. The above diets and drugs were fed throughout the 8 weeks. At 16 weeks old, the rats were euthanized under general anesthesia. This study was approved by the Korea University Institute of Animal Care and Use Committee.

Reverse transcriptase-polymerase chain reaction. Bone marrow cells were collected from the femurs of the S-D rats. After washing with PBS three times, the cells were diluted with a $1 \mathrm{ml}$ solution including $50 \mathrm{mM}$ HEPES (pH 7.0), $150 \mathrm{mM}$ $\mathrm{NaCl}, 1.5 \mathrm{mM} \mathrm{MgCl}, 1 \%$ Triton, $10 \mathrm{mM}$ sodium pyrophosphate, $1 \mathrm{mMPMSF}$ (Sigma-Aldrich, St. Louis, MO,USA) and $10 \mu \mathrm{g} / \mathrm{ml}$ leupeptin (Sigma-Aldrich). After mixing and centrifugation with $500 \mu 1$ TRIzol solution (Invitrogen, Grand Island, NY, USA) and $100 \mu \mathrm{l}$ chloroform, supernatants from the acquired cell extracts were used for the reverse transcriptase-polymerase chain reaction (RT-PCR). After washing the supernatants with $75 \%$ ethanol, the total RNA was extracted, and the reaction mixture composed of $10 \mathrm{X}$ reverse transcription buffer, RNase inhibitor, dNTP, M-MLV reverse transcriptase and $1 \mu \mathrm{l}$ total RNA was incubated at $42^{\circ} \mathrm{C}$ for $15 \mathrm{~min}$ to synthesize cDNA. The cDNA produced was amplified into PCR using sense/antisense primers (Table I). The resulting product was dissolved in $2 \%$ agarose gel, dyed with ethidium bromide and developed under UV light. To qualify the reactants, the band was measured using densitometry and each ratio to GAPDH was calculated.

Measurement of serum CTX-1. After sacrificing the experimental S-D rats, their blood was sampled from the right atrium. Serum was produced by centrifugation of the collected blood. An immunoassay kit (RatLaps ${ }^{\mathrm{TM}}$, ImmunoDiagnostic Systems, Herlev, Denmark) was used for the measurement of CTX-1. In brief, $100 \mu \mathrm{l}$ of biotinylated RatLaps antigen (Ag biotin) was added to each well, incubated for $30 \pm 5 \mathrm{~min}$ at room temperature, the immuno strips were washed manually five times using $300 \mu \mathrm{l}$ of washing solution (wash buffer) 


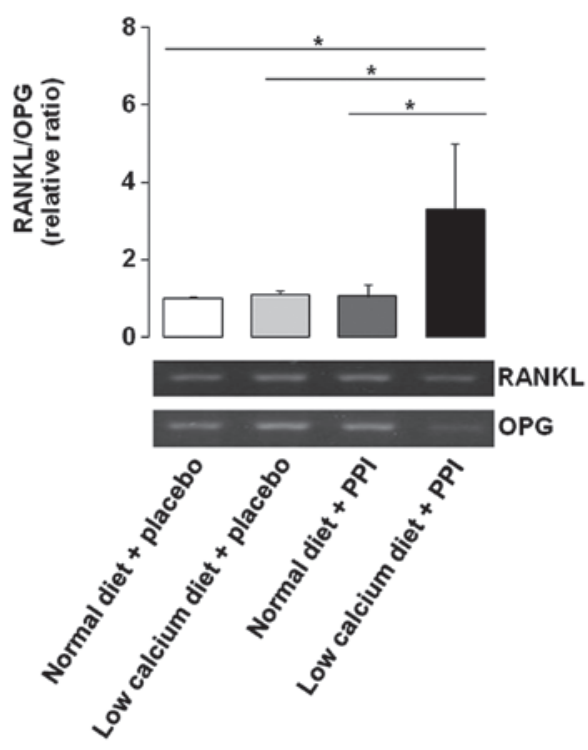

Figure 1. Relative ratio of RANKL/OPG by RT-PCR. RANKL/OPG ratio is significantly increased by low calcium diet and PPI, compared with the other three groups. GAPDH mRNA levels are shown as an internal control. Error bars show standard deviation of mean values. RANKL, receptor activator of NF-kB ligand; OPG, osteoprotegerin; PPI, proton pump inhibitor. " $\mathrm{P}<0.05 ; \mathrm{n}=4$ for each group.

and $20 \mu \mathrm{l}$ serum samples were added from each group into the appropriate wells followed by $100 \mu 1$ of primary antibody. Samples were incubated overnight $(18 \pm 3 \mathrm{~h})$ at $2-8^{\circ} \mathrm{C}$ and washed a further five times. Following the addition of $100 \mu \mathrm{l}$ of peroxidase-conjugated goat anti-rabbit IgG antibody (enzyme conjugate) to each well, samples were incubated for $60 \pm 5 \mathrm{~min}$ at room temperature, $100 \mu \mathrm{l}$ of substrate solution was added to each well and incubated for $15 \pm 2 \mathrm{~min}$ at room temperature in the dark and $100 \mu \mathrm{l}$ of stopping solution was then added to each well. Finally, the absorbance was measured within $2 \mathrm{~h}$ at $450 \mathrm{~nm}$, with a reading at $650 \mathrm{~nm}$ acting as a reference.

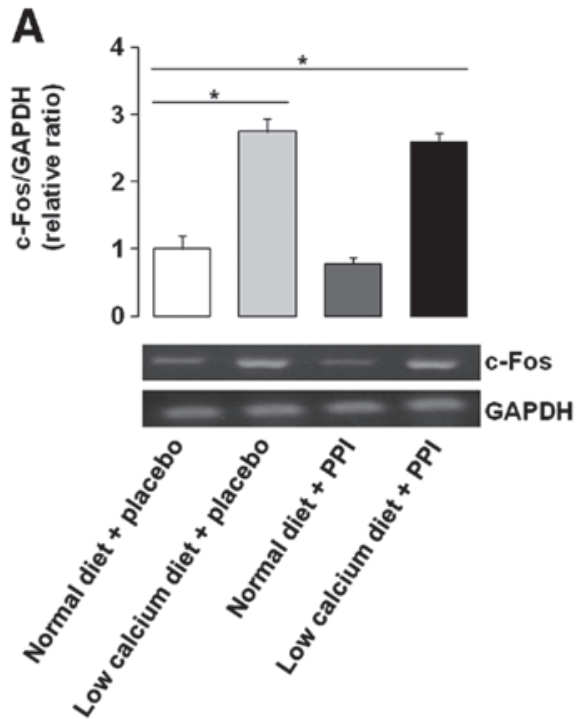

Measurement of serum calcium and alkaline phosphatase levels. Whole blood was collected and serum was obtained using the same method as that mentioned previously. Serum levels of calcium and alkaline phosphatase (ALP) were measured by activity analysis using a reagent $\left(\right.$ ELITech $^{\mathrm{TM}}$, Provence, France).

Statistical analysis. SPSS for Windows (version 12.0, SPSS, Inc., Chicago, IL, USA) was used as the statistical analysis program. Continuous variables were analyzed using ANOVA when the variables followed a normal distribution, and the Kruskal-Wallis test (a non-parametric method) when they did not follow a normal distribution. $\mathrm{P}<0.05$ was considered to indicate a statistically significant difference.

\section{Results}

RANKL/OPG ratio is increased by the combination of a low calcium diet and PPI. To evaluate the relative ratio of RANKL/OPG, the RT-PCR method was performed. Initially, the gene expression level of RANKL was upregulated by low calcium diet alone and PPI alone, compared with normal diet alone. Expression levels of OPG were also upregulated by PPI alone, compared to that of normal diet alone, which showed a similar pattern to RANKL. However, OPG was markedly downregulated by a low calcium diet with PPI. Consequently, the RANKL/OPG ratio was significantly increased by a low calcium diet with PPI, compared to the other three groups (Fig. 1). Given that OPG binds competitively to RANKL to inhibit RANKL-RANK binding, if the administration of PPI is combined with a low calcium diet, it may enhance the signal transduction through RANK.

Gene expression of c-Fos and NFATcl is upregulated by low calcium diet with or without PPI. Relative mRNA expression levels of c-Fos, a transcription factor of the RANKL/ RANK/NF- $\mathrm{B}$ signaling pathway, was also measured by

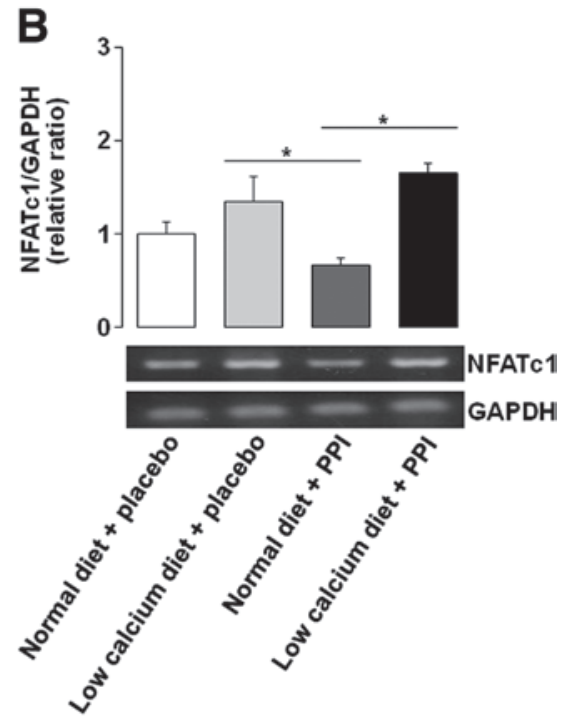

Figure 2. Gene expression level of c-Fos and NFATc1. (A) c-Fos is significantly increased by low calcium diet with/without PPI, compared with normal diet alone. (B) NFATc1 is significantly increased by low calcium diet with/without PPI, compared with PPI alone. Error bars represent standard deviation of mean values. NFATc1, nuclear factor of activated $\mathrm{T}$ cell $\mathrm{c} 1$; PPI, proton pump inhibitor. ${ }^{*} \mathrm{P}<0.05 ; \mathrm{n}=4$ for each group. 

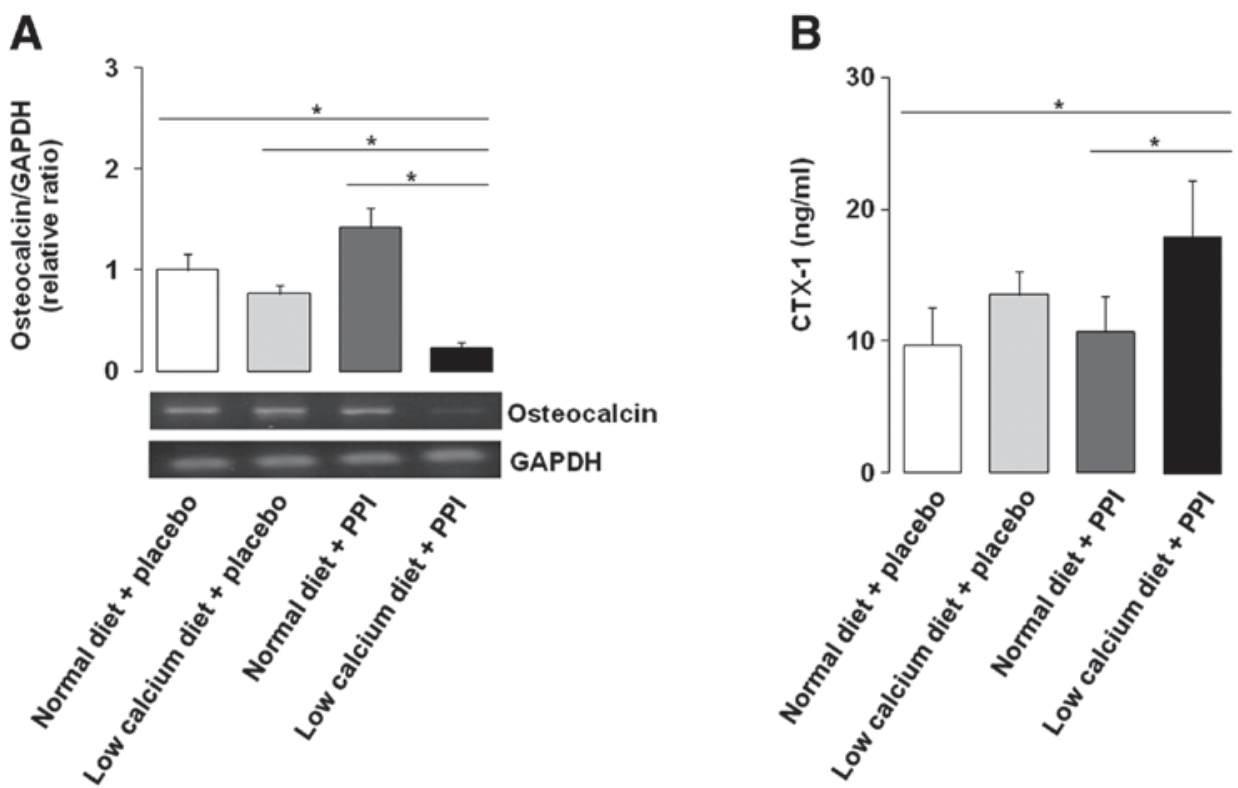

Figure 3. Measurement of osteocalcin and CTX-1. (A) Relative gene expression of osteocalcin is downregulated by a combination of PPI and low calcium diet, compared with the other three groups. (B) Serum calcium level of CTX-1 was also significantly increased by PPI with low cacium diet. Representative band for osteocalcin is shown. GAPDH mRNA levels are shown as an internal control. Error bars represent standard deviation of mean values. CTX-1, C-terminal crosslinking telopeptide of type I; PPI, proton pump inhibitor. " $\mathrm{P}<0.05 ; \mathrm{n}=4-5$ for each group.
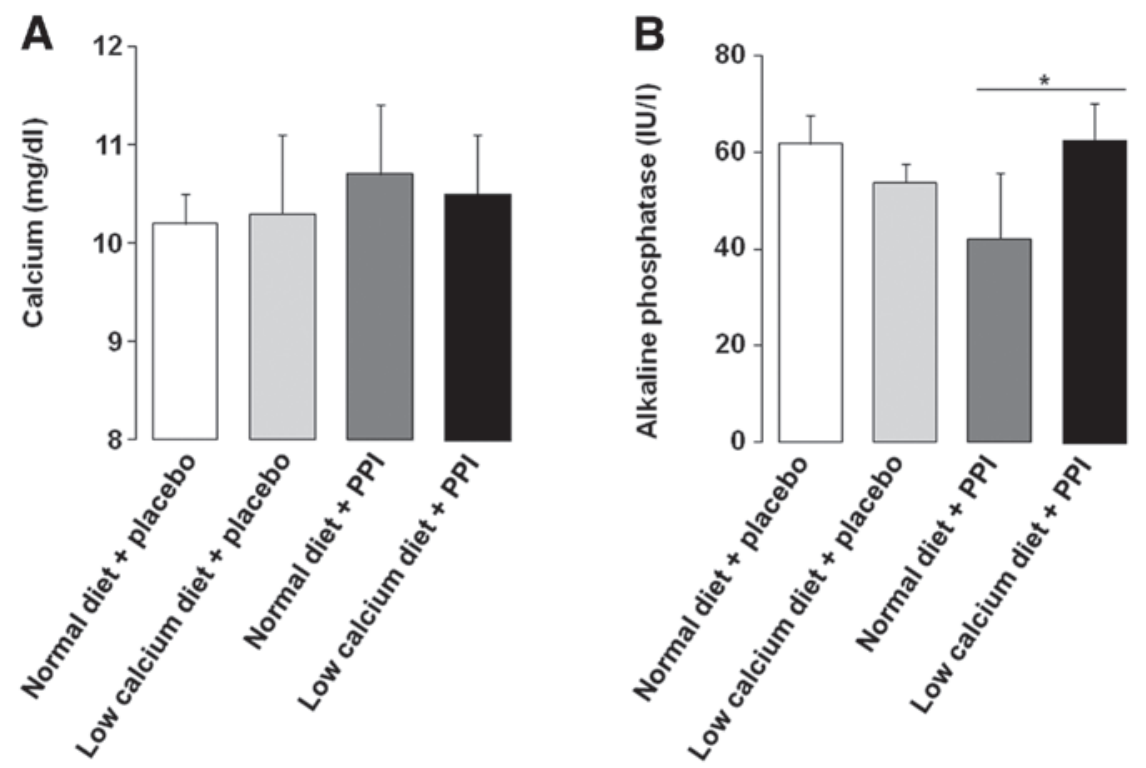

Figure 4. Measurement of serum calcium and ALP. (A) The calcium level was not significantly different between the four groups. (B) ALP level was increased by low calcium with PPI administration, compared with PPI alone. Error bars represent standard deviation of mean values. ALP, alkaline phosphatase; PPI, proton pump inhibitor. ${ }^{*} \mathrm{P}<0.05 ; \mathrm{n}=5$ for each group.

the RT-PCR method. C-Fos levels were increased by a low calcium diet with or without PPI, compared with that of a normal diet alone, by 131 and $115 \%$, respectively (Fig. 2A). NFATc1 is a target gene for c-Fos activation, and showed a similar pattern for c-Fos expression, which was increased by a low calcium diet with or without PPI, compared with that of PPI alone, by 87 and 107\%, respectively (Fig. 2B). When considered together with the RANKL/OPG ratio, it is suggested that PPI alone may not cause differentiation and activation of osteoclasts, as the autoamplification of NFATc1 mediates completion of the differentiation process as the final step to several signaling pathways (20).

Osteocalcin is decreased and CTX-1 is increased by the combination of a low calcium diet and PPI. Relative mRNA expression levels of osteocalcin, a well-known bone formation biochemical marker, were significantly downregulated by a low calcium diet with PPI, compared with the other 
three groups, by $-83,-80$ and $-89 \%$, respectively (Fig. 3A). By contrast, the bone resorption marker serum CTX-1 was significantly higher in the group with a low calcium diet and PPI compared with the normal diet alone or PPI alone groups (Fig. 3B). It is suggested that PPI, together with a low calcium diet, may induce alteration of macro- or micro-structure and contribute to the pathogenesis of bone metabolism.

Serum concentration of calcium and ALP. Serum calcium levels were not significantly different among the four groups (Fig. 4A). However, the serum ALP level was found to be significantly higher in the group with a low calcium diet and PPI, compared with PPI alone (Fig. 4B). Generally, serum calcium levels do not change greatly beyond the normal limit despite ovariectomization in the rat model. Our result is thought to be consistent with previous data, which suggested that serum calcium remains constant under internal or external experimental conditions $(12,26)$. Considering that serum ALP usually acts as an early biomarker of osteoblast differentiation and bone formation (27), the difference in serum ALP in our study conflicts with that of osteocalcin, which was markedly decreased by the low calcium diet with PPI. However, we believe that the osteocalcin result in our study is more specific in reflecting the alteration to bone formation than that of serum ALP, due to the fact that we used extracts taken directly from femoral bone marrow cells for the analysis of the osteocalcin activity.

\section{Discussion}

In this in vivo study we aimed to show how PPI leads to bone turnover and to provide an explanation of the exact mechanism. Based on clinical data suggesting that PPI increased fracture risk in a duration- or dose-dependent manner, we induced the long-term effects of PPI by administering $30 \mathrm{mg} / \mathrm{kg}$ for 8 weeks, which was longer than in previous rat model studies where rats ingested omeprazole $20-30 \mathrm{mg} / \mathrm{kg}$ for 4 days to 3 weeks (28-30).

In this study, we evaluated the effect of PPI on differentiation and activation of osteoclasts and bone resorption. RANKL/RANK binding and its sequential cascade of c-Fos and NFATc1 was not increased in the group with a regular diet and PPI. By contrast, the relative ratio of RANKL/OPG and mRNA levels of c-Fos and NAFATc1 were increased by the combination of a low calcium diet and PPI administration, and we suggest that osteoclast activity may be enhanced by the combination of a low calcium diet and PPI. Such consideration is supported by the finding that serum CTX-1 levels, a reliable bone resorption marker, were also increased with a low calcium diet and PPI, compared with a regular diet with or without PPI administration. Another notable finding is that the expression level of osteocalcin was reduced significantly by the combination of a low calcium diet with PPI compared with the other three groups. However, it is controversial as to whether low calcium diet and PPI have a synergic effect on the activation of osteoclasts and the inhibition of osteoblasts. Experimental data supporting the hypothesis for bone resorption or formation is still lacking, and further studies are required.

We should be cautious about interpreting the outcomes of this study after the consideration of several aspects. Firstly, from the point of osteoclasts, there is a limit to the direct comparison of the effect of omeprazole from that of other synthetic V-ATPase inhibitors (i.e., FR167356, FR202126, SB242784), which showed inhibitory effects on bone resorption and activation of osteoclasts in previous in vivo studies $(14,26)$, as their potency on the V-ATPase of an osteoclast may be different. Secondly, PPI can be a double-edged sword in reducing intestinal absorption of calcium or inhibiting activation of osteoclasts, and profound acid suppression with high-dose and long-term PPI therapy may be necessary to have a significant impact on bone metabolism (10). Thirdly, it would have been helpful in supporting our data if we had scanned images from more than two sites by micro-CT, in order to reveal alterations to trabecular microstructure. Previous studies using ovariectomized rats reported that significant changes of trabecular microstructures were detected from both the femur neck and vertebral body by micro-CT $(31,32)$.

In conclusion, our results suggest that the combination of a low calcium diet with PPI produces a possible synergic effect on bone resorption, and calcium supplements are necessary when a patient takes PPIs for a long time. Further studies are required in the near future to investigate whether their synergism clinically affects bone resorption and increases the risk of osteoporosis and pathologic fracture.

\section{References}

1. El-Serag HB: Time trends of gastroesophageal reflux disease: a systematic review. Clin Gastroenterol Hepatol 5: 17-26, 2007.

2. DeVault KR and Castell DO: Updated guidelines for the diagnosis and treatment of gastroesophageal reflux disease. Am J Gastroenterol 100: 190-200, 2005.

3. Soll AH: Consensus conference. Medical treatment of peptic ulcer disease. Practice guidelines. Practice Parameters Committee of the American College of Gastroenterology. JAMA 275: 622-629, 1996.

4. Talley NJ and Vakil N: Guidelines for the management of dyspepsia. Am J Gastroenterol 100: 2324-2337, 2005.

5. van Zanten SV, Armstrong D, Chiba N, et al: Esomeprazole 40 mg once a day in patients with functional dyspepsia: the randomized, placebo-controlled 'ENTER' trial. Am J Gastroenterol 101: 2096-2106, 2006.

6. Becker V, Bajbouj M, Waller K, Schmid RM and Meining A: Clinical trial: persistent gastro-oesophageal reflux symptoms despite standard therapy with proton pump inhibitors - a follow-up study of intraluminal-impedance guided therapy. Aliment Pharmacol Ther 26: 1355-1360, 2007.

7. Dent J, Brun J, Fendrick A, et al: An evidence-based appraisal of reflux disease management - the Genval Workshop Report. Gut 44 (Suppl 2): S1-S16, 1999.

8. Jacobson BC, Ferris TG, Shea TL, Mahlis EM, Lee TH and Wang TC: Who is using chronic acid suppression therapy and why? Am J Gastroenterol 98: 51-58, 2003.

9. Targownik LE, Lix LM, Metge CJ, Prior HJ, Leung S and Leslie WD: Use of proton pump inhibitors and risk of osteoporosis-related fractures. CMAJ 179: 319-326, 2008.

10. Yang YX, Lewis JD, Epstein S and Metz DC: Long-term proton pump inhibitor therapy and risk of hip fracture. JAMA 296: 2947-2953, 2006.

11. Chonan O, Takahashi R, Yasui H and Watanuki M: Effect of L-lactic acid on calcium absorption in rats fed omeprazole. J Nutr Sci Vitaminol 44: 473-481, 1998

12. O'Connell MB, Madden DM, Murray AM, Heaney RP and Kerzner LJ: Effects of proton pump inhibitors on calcium carbonate absorption in women: a randomized crossover trial. Am J Med 118: 778-781, 2005.

13. Karsdal MA, Henriksen K, Sorensen MG, et al: Acidification of the osteoclastic resorption compartment provides insight into the coupling of bone formation to bone resorption. Am J Pathol 166: 467-476, 2005 
14. Niikura K, Takeshita $\mathrm{N}$ and Takano M: A vacuolar ATPase inhibitor, FR167356, prevents bone resorption in ovariectomized rats with high potency and specificity: potential for clinical application. J Bone Miner Res 20: 1579-1588, 2005.

15. Kocsis I, Arato A, Bodánszky H, et al: Short-term omeprazole treatment does not influence biochemical parameters of bone turnover in children. Calcif Tissue Int 71: 129-132, 2002.

16. Mizunashi K, Furukawa Y, Katano K and Abe K: Effect of omeprazole, an inhibitor of $\mathrm{H}^{+}, \mathrm{K}(+)$-ATPase, on bone resorption in humans. Calcif Tissue Int 53: 21-25, 1993.

17. Suda T, Takahashi N, Udagawa N, Jimi E, Gillespie MT and Martin TJ: Modulation of osteoclast differentiation and function by the new members of the tumor necrosis factor receptor and ligand families. Endocr Rev 20: 345-357, 1999.

18. Boyce BF and Xing L: Functions of RANKL/RANK/OPG in bone modeling and remodeling. Arch Biochem Biophys 473: $139-146,2008$.

19. Huang H, Ryu J, Ha J, et al: Osteoclast differentiation requires TAK1 and MKK6 for NFATc1 induction and NF-kappaB transactivation by RANKL. Cell Death Differ 13: 1879-1891, 2006.

20. Boyce BF and Xing L: The RANKL/RANK/OPG pathway. Curr Osteoporos Rep 5: 98-104, 2007.

21. Kwan Tat S, Pelletier JP, Lajeunesse D, Fahmi H, Lavigne M and Martel-Pelletier J: The differential expression of osteoprotegerin (OPG) and receptor activator of nuclear factor kappaB ligand (RANKL) in human osteoarthritic subchondral bone osteoblasts is an indicator of the metabolic state of these disease cells. Clin Exp Rheumatol 26: 295-304, 2008.

22. Matsuo K, Galson DL, Zhao C, et al: Nuclear factor of activated T-cells (NFAT) rescues osteoclastogenesis in precursors lacking c-Fos. J Biol Chem 279: 26475-26480, 2004.

23. Takayanagi H, Kim S, Koga T, et al: Induction and activation of the transcription factor NFATc1 (NFAT2) integrate RANKL signaling in terminal differentiation of osteoclasts. Dev Cell 3: 889-901, 2002.
24. Yamashita T, Yao Z, Li F, et al: NF-kappaB p50 and p52 regulate receptor activator of NF-kappaB ligand (RANKL) and tumor necrosis factor-induced osteoclast precursor differentiation by activating c-Fos and NFATc1. J Biol Chem 282: 18245-18253, 2007.

25. Lee BJ, Park JJ, Joo MK, et al: Effect of proton pump inhibitor on the bone turnover in the ovariectomized ICR mice. J Gastroenterol Hepatol 23 (Suppl 5): A10, 2008.

26. Niikura K, Takeshita $\mathrm{N}$ and Chida N: A novel inhibitor of vacuolar ATPase, FR202126, prevents alveolar bone destruction in experimental periodontitis in rats. J Toxicol Sci 30: 297-304, 2005.

27. Sorensen MG, Henriksen K, Schaller S and Karsdal MA: Biochemical markers in preclinical models of osteoporosis. Biomarkers 12: 266-286, 2007.

28. Cadir FO, Bicakci U, Tander B, et al: Protective effects of vitamin $\mathrm{E}$ and omeprazole on the hypoxia/reoxygenation induced intestinal injury in newborn rats. Pediatr Surg Int 24: 809-813, 2008.

29. Diogo Filho A, Santos PS, Duque AS, Cezário RC and Gontijo Filho PP: Experimental model in the qualitative and quantitative assessment of non-Helicobacter gastric microflora under proton pump inhibitors action. Acta Cir Bras 21: 279-284, 2006.

30. Elseweidy MM, Younis NN, Amin RS, Abdallah FR, Fathy AM and Yousif ZA: Effect of some natural products either alone or in combination on gastritis induced in experimental rats. Dig Dis Sci 53: 1774-1784, 2008.

31. Otomo H, Sakai A, Ikeda S, et al: Regulation of mineral-to-matrix ratio of lumbar trabecular bone in ovariectomized rats treated with risedronate in combination with or without vitamin $\mathrm{K} 2$. J Bone Miner Metab 22: 404-414, 2004.

32. Song YH, Lee W, Lee CJ, Ji JH and Lee BD: Study of bony trabecular characteristics using bone morphometry and micro-CT. Korean J Oral Maxillofac Radiol 37: 27-33, 2007. 